Service social

\title{
La drogue, front de lutte pour une société alternative?
}

\section{Giulio Girardi}

Volume 39, numéro 2, 1990

Les problèmes sociaux

URI : https://id.erudit.org/iderudit/706479ar

DOI : https://doi.org/10.7202/706479ar

Aller au sommaire du numéro

Éditeur(s)

École de service social de l'Université Laval

ISSN

1708-1734 (numérique)

Découvrir la revue

Citer cet article

Girardi, G. (1990). La drogue, front de lutte pour une société alternative? Service social, 39(2), 102-114. https://doi.org/10.7202/706479ar

\section{Résumé de l'article}

L'auteur aborde le problème de la drogue dans une perspective sociologique qui met en relation facteurs structurels et facteurs culturels : marchéde la drogue, société capitaliste, toxicomanie, projet de société. Il émet l'hypothèse que la lutte contre la drogue doit, pour être efficace, s'insérer dans une recherche d'alternative de société. Il donne comme exemple de cette recherche l'expérience de certaines communautés d'accueil, en Italie, et résume les principes qui guident leur action. 
Giulio Girardi, professeur à I'Université de Sassari, Italie'.

Traduit de l'italien, avec la permission de l'auteur, par Gérald Doré, professeur à l'École de service social de l'Université Laval'2.

\section{La drogue, front de lutte pour une société alternative?}

Giulio Girardi

Dans le débat bruyant qui s'est ouvert en Italie sur la lutte contre la drogue, un des aspects les plus déconcertants est le caractère répétitif d'une grande partie des interventions, en particulier celles qui viennent de l'Hôtel du Gouvernement. Les différentes mesures proposées, quelquefois avec des accents messianiques, pour « abattre le fléau ", ont toutes été largement expérimentées, en Italie et ailleurs, et se sont révélées non seulement inefficaces, mais souvent contre-productives. Un débat ainsi lancé est en réalité une préoccupante déclaration d'impuissance.

Mais peut-être une tentative de renouveler la réflexion sur le sujet pourrait-elle précisément partir de cette constatation, en s'interrogeant justement sur les raisons de cette impuissance, de ces échecs, de cette répétitivité. C'est en réponse à cette question que je voudrais avancer une hypothèse : la raison principale pour laquelle notre société se découvre impuissante face au phénomène de la drogue, aussi bien au niveau du trafic qu'à celui de la consommation, est que celui-ci plonge ses racines dans la logique même du système capitaliste, dans ses structures et sa culture; c'est pourquoi la société ne peut affronter sérieusement le problème sans se remettre éllemême en cause.

Si cette analyse se révélait valable, elle contiendrait aussi une indication sur la piste à suivre pour combattre efficacement la drogue : à savoir la nécessité d'inclure cette lutte dans un projet global d'alternative sociale. Mais ne serait-ce pas là une nouvelle façon d'avouer sa propre impuissance, étant donné que le «projet global d'alternative sociale » a un caractère utopique, c'est-à-dire n'a aucune probabilité d'être réalisé, du moins dans un avenir prévisible ? C'est précisément le problème que je voudrais aborder, en 
avançant une seconde hypothèse : à savoir qu'une contribution très significative au renouvellement de la recherche, et par conséquent de l'action, sur ce terrain, pourrait venir de quelques communautés d'accueil; de celles justement qui se caractérisent par leur tentative de relier le problème de la drogue à celui de la société, l'alternative à la drogue à l'alternative de société ${ }^{3}$.

Je développerai ces hypothèses, en réfléchissant sur les rapports qui lient à la société capitaliste le marché de la drogue, la toxicomanie et des formes déterminées de lutte à la drogue.

\section{Marché de la drogue et société capitaliste}

La drogue est aujourd'hui une des marchandises les plus lucratives, sinon la plus lucrative, qui permet, sur le plan international, une énorme accumulation de capital et, par conséquent, de pouvoir. Elle est de plus une marchandise d'un type particulier, puisque illégale, qui est échangée sur un marché clandestin, où l'économie s'entremêle avec le crime organisé. Elle est aussi d'un type particulier, parce que la réponse qu'elle apporte au besoin de gratification et de détente psychique est, à long terme, illusoire : son effet réel est, au bout du compte, l'inverse de celui attendu par le consommateur.

Ces caractéristiques de la marchandise-drogue ne la repoussent cependant pas sur les marges du marché. Elles en accentuent plutôt la connaturalité avec le système capitaliste, en faisant d'elle un révélateur de sa logique, qui opère sur le plan légal sous un mode moins visible : logique axée sur la lutte pour la vie dominée par la survivance du plus fort. L'illégalité du marché multiplie en fait la valeur d'échange de la marchandise et, par conséquent, les profits qu'elle permet. Elle rend plus absolue et despotique la domination du capitalisme, éliminant même les contrôles et les limitations qui, dans un contexte de légalité, peuvent lui imposer quelque frein.

Les capitaux accumulés à travers le marché de la drogue se trouvent par la suite habituellement réinvestis dans des opérations légales, ce qui provoque un entrelacement inextricable entre les deux volets de l'activité économique, entrelacement qui marque en profondeur, sur le plan international, le système en son entier. Dans celui-ci, le marché occulte assume une importance croissante, et tend à transférer sur le marché légal ses propres méthodes.

Non moins profond est le lien que la drogue entretient avec la logique capitaliste de par le caractère illusoire de la réponse qu'elle apporte à un besoin. De ce point de vue, elle exprime en fait, de la manière la plus convaincante, la nature " fétichiste » de la marchan- 
dise, telle que brillamment illustrée par Marx : à savoir le fait que la valeur d'échange qui lui est attribuée est indépendante de sa nature et de son efficacité réelles, tout en étant une illusion générée par des rapports réels de domination.

La drogue présente en outre des affinités avec une autre marchandise éminemment lucrative, les armes. Comme elles, la drogue appartient au marché de la mort. Comme les armes, elle met à nu la logique capitaliste suivant laquelle la croissance des uns est alimentée par le sang des autres, la vie des uns par la mort des autres.

Mais il y a un autre aspect important du commerce de la drogue, qui le lie étroitement au système capitaliste, et c'est son rapport au sous-développement. En effet, presque tous les pays qui produisent les matières premières utilisées pour la préparation des drogues sont sous-développés et réduits à une économie de survie. Pour les producteurs, la drogue n'est pas une source de gains fabuleux, mais une condition de subsistance. Or, le sous-développement, qui fait de la production de la drogue une nécessité vitale pour ces pays, est un produit du développement capitaliste des pays riches.

La mafia, qui gère dans une large mesure ce trafic, est habituellement perçue comme un phénomène de déviance, pathologique, lié à des conditions particulières d'arriération sociale et culturelle et, par conséquent, en opposition à une société vue comme fondamentalement saine.

Mais si les hypothèses ici ébauchées sont valides, la mafia serait au contraire connaturelle au système capitaliste et contribuerait de manière déterminante à caractériser, sur le plan international, sa phase actuelle. Cette connaturalité se manifeste dans l'interpénétration des opérations économiques illégales du crime organisé et de ses opérations légales. Parmi les activités économiques illégales et légales, l'activité financière assume une importance croissante, plus précisément le mécanisme du système bancaire international à travers lequel l'argent sale est recyclé. La "mafiosité » n'est donc pas seulement caractéristique de quelques multinationales, mais dans une certaine mesure, de toutes, même s'il s'en trouve qui en expriment la logique avec moins de scrupules et de manière plus conséquente.

Si ce qui précède est vrai, le marché de la drogue est si connaturel à l'économie capitaliste que son avenir apparaît intimement lié à celui du système. Une stratégie réaliste de lutte à la drogue doit, par conséquent, tenir compte de ce fait : la drogue est retranchée dans une forteresse, l'économie capitaliste, et c'est seulement en attaquant cette forteresse qu'il sera possible de l'atteindre.

Le marché de la drogue n'est pas seulement un phénomène de première importance sur le plan économique, mais il a de nombreu- 
ses conséquences sur le plan politique. Les profits fabuleux qu'il procure sont de par leur nature, au niveau international, la source d'un énorme pouvoir occulte. Ils permettent aux multinationales de la drogue et du crime d'influencer les organisations politiques, d'établir des alliances avec des personnalités en vue, d'obtenir l'appui de l'État à leurs activités " légales » et de garantir l'impunité à celles qui sont illégales. C'est précisément cette interconnexion clandestine entre crime organisé, économie illégale et légale, et manipulation politique, qui caractérise le pouvoir de la mafia. Par ce biais, celle-ci tend à devenir, et selon toute probabilité est déjà devenue, sur le plan national et international, partie intégrante du bloc dominant.

L'influence de la mafia sur la vie politique en accélère la corruption et pour cette raison même, l'évolution dans une direction autoritaire : ses opérations doivent en effet, pour prospérer, être soustraites à quelque contrôle démocratique que ce soit. Transférant ensuite sur le terrain politique les méthodes criminelles expérimentées sur le plan économique, elle favorise le déploiement d'une vie politique déliée de toute règle morale et dominée, comme l'économie, par la loi de la jungle. En réalité, encore ici la drogue, dans les mains de la mafia, ne fait qu'exalter et rendre plus manifeste une logique qui, de fait, domine la vie politique, nationale et internationale. La bataille contre le trafic de la drogue devient, par conséquent, partie intégrante de la bataille nationale et internationale pour la démocratie.

\section{Toxicomanie et société capitaliste}

Si d'un côté, la société capitaliste favorise le marché de la drogue, de l'autre, elle contribue à susciter avec la toxicomanie, le besoin auquel le marché doit répondre. De ce double point de vue, elle est toxicogène.

Certes, la toxicomanie n'est un phénomène exclusif ni à l'époque contemporaine ni à la société capitaliste; mais il y a un type de toxicomanie qui est l'expression de cette société, dans la phase actuelle de son développement. Je veux dire que la toxicomanie est un phénomène historique qui ne révèle sa signification qu'en rapport à son contexte social. La toxicomanie qu'il nous intéresse d'analyser est précisément celle qui, au cours des dernières décennies, dans les sociétés du capitalisme avancé, a pris des dimensions de masse, au point de s'imposer comme un des problèmes sociaux les plus graves de l'époque.

Observée de près, la toxicomanie révèle un rapport organique avec cette société. On le comprend en analysant les itinéraires des 
jeunes vers la drogue, en se demandant ce qu'ils y cherchent, d'où provient le pouvoir de séduction qu'elle exerce sur eux. Or, beaucoup de jeunes, sinon tous, rencontrent la drogue à l'intérieur d'une recherche sur le sens de la vie, recherche qui n'a pas trouvé de réponse d'ailleurs. Si ceci est vrai, comprendre leur itinéraire veut dire identifier les raisons pour lesquelles cette recherche a échoué.

Cette recherche n'a pas cependant un caractère "métaphysique " mais historique. Elle consiste en effet à prendre position face aux perspectives d'avenir, au système de valeurs, aux idéaux, proposés par la société, à travers la famille, l'école, l'Église, les moyens de communication de masse, le marché du travail. Or précisément, dans ce contexte, le recours à la drogue exprime une contestation radicale, un refus, une recherche alternative.

En cela même, elle devient expression dramatique d'un conflit de générations, d'une révolte des jeunes contre les adultes, des enfants contre les parents. II ne s'agit pas pourtant du conflit récurrent entre les générations, analysé en termes généraux par les psychologues et les psychanalystes, mais d'un conflit spécifique, lié à la situation historique actuelle, conflit caractérisé par une accélération sans précédent des transformations structurelles et culturelles, et par une distanciation conséquente entre les générations.

Dans quelques itinéraires vers la drogue, ce contenu social et politique du message est explicite. Ainsi, une génération de toxicomanes "soixante-huitards " a vécu et théorisé le recours à la drogue comme contestation du système, transgression de ses normes, refus de ses valeurs, recherche d'une communauté et d'un horizon différents. "Se piquer " était pour eux un geste chargé de symbolisme révolutionnaire.

Pour beaucoup ensuite, le recours à la drogue est devenu un besoin plus impérieux, suite aux frustrations politiques et personnelles suscitées par les défaites, le désenchantement, la chute des mythes et des espérances. La drogue était alors un cri de désespoir; analogue peut-être à cette option du suicide dans laquelle tant de militants exprimèrent leur refus d'un monde se révélant irrémédiablement absurde.

Mais aujourd'hui, pour la majorité des toxicomanes, l'interrelation entre drogue et politique n'est pas aussi explicite. Leur recherche de sens se présente en termes plus strictement personnels. Même pour eux, cependant, cette recherche se situe objectivement dans un contexte social et culturel déterminé, à la lumière duquel elle doit être interprétée : les toxicomanes eux-mêmes le font d'ailleurs entre eux avec beaucoup de lucidité quand, ayant décidé d'arrêter, ils s'engagent sur la route difficile de la conscientisation et de la libération. 
Pour saisir le sens de leur itinéraire vers la drogue, ils s'interrogent alors sur l'origine de cette insatisfaction profonde, de cette inquiétude, de ce sentiment de solitude, qui l'a amorcé : cet état intérieur n'est pas provoqué, disent plusieurs, par un manque de sécurité économique, de bien-être, de travail, mais plutôt par un manque de sens, de raison de vivre.

II n'est pas difficile de saisir les racines sociales de cette insatisfaction, la charge de contestation politique latente en elle. La société capitaliste avancée est en effet caractérisée par une coupure majeure entre les aspirations qu'elle suscite, au niveau des masses, et les possibilités réelles qu'elle offre de les satisfaire. Elle suscite chez tous ses membres, et particulièrement chez les jeunes, à travers la promotion culturelle, les moyens de communication de masse et la publicité, des aspirations très hautes. Par ailleurs, elle est organisée économiquement, politiquement et culturellement, au service de minorités privilégiées, de telle sorte qu'elle rend objectivement impossible pour la grande majorité la satisfaction de ces aspirations. Le système est, par conséquent, structuré de manière à générer de la frustration au niveau des masses, surtout parmi les jeunes.

Cet écart entre aspirations et possibilités de les satisfaire est rendu plus stressant et angoissant par le système de valeurs dans lequel les jeunes sont habituellement formés, système inspiré par une conception individualiste et compétitive de la vie : où le succès sur le terrain scolaire, sportif, sexuel, économique, professionnel, culturel, etc., consiste à l'emporter sur les autres, à être le premier. C'est précisément ce climat qui engendre fatalement de profondes frustrations, soit chez les vaincus, soit encore chez les vainqueurs qui, au milieu du succès, découvrent que celui-ci ne satisfait pas leurs attentes les plus vraies.

Par des chemins différents mais convergents donc, le recours à la drogue se révèle une tentative désespérée de résoudre le problème du sens de la vie. Désespérée, parce qu'elle dénonce l'inconsistance des réponses courantes et le vide qu'elles n'ont pas réussi à combler; désespérée, parce qu'elle exprime la protestation contre un type de vie, de société, de culture, auquel par ailleurs on ne voit pas d'alternatives possibles.

La drogue est, par conséquent, une fuite, mais aussi une dénonciation de l'absurdité du système; une fuite, mais qui exprime, à sa manière, une recherche anxieuse de vie, d'alternative de communication, d'amour. Dans un monde illusoire, parce que dans le monde réel, tout ceci s'est révélé impossible.

Si ces analyses sont valables, la toxicomanie sera certes une déviance; mais beaucoup plus profonde et mortelle est la déviance qu'elle dénonce, celle $d^{\prime}$ un système qui confisque la richesse, le 
pouvoir, la culture, la vie, au bénéfice de minorités, en en excluant de grandes masses d'hommes et de femmes. Un vol, celui-ci, beaucoup plus coupable que tous ceux que les drogués seront poussés à faire pour se procurer le "stock". La toxicomanie devient alors un appel lancé par les jeunes aux adultes, pour qu'ils s'interrogent plus lucidement, plus honnêtement, plus courageusement, sur le sens du progrès qu'ils poursuivent et de l'avenir qu'ils préparent, sur la valeur de cette "normalité " qu'ils prétendent imposer aux jeunes " déviants".

\section{Société capitaliste et riposte à la drogue}

La difficulté fondamentale de la lutte à la drogue est précisément celle-ci : notre société se trouve à devoir combattre un "fléau " qu'elle-même engendre, avec ses structures et sa culture. Elle doit, par conséquent, le combattre sans pouvoir en toucher les causes, parce que ceci impliquerait une radicale autocritique, une remise en question du système lui-même. Elle doit, au contraire, le combattre sans même pouvoir l'analyser sans préjugés, parce que la culture dominante fait écran entre les détenteurs du pouvoir et la réalité sociale : cette culture est elle-même en effet une drogue lourde pleinement légalisée, qui obnubile la vision de ceux qui en sont pénétrés. C'est cette "drogue légale» qui est l'obstacle le plus sérieux à une lutte efficace contre les autres drogues.

Une telle contradiction explique aussi pourquoi jusqu'à maintenant les mesures adoptées, sur le plan international, pour combattre la drogue, se sont avérées inefficaces, contre-productives même : en particulier le prohibitionnisme, la criminalisation et la pénalisation des toxicomanes, le recours à des formes coercitives de traitement.

Ainsi le prohibitionnisme a servi jusqu'à maintenant beaucoup plus à favoriser le marché de la drogue qu'à le décourager: les avantages qu'il apporte au trafic, en multipliant la valeur d'échange de la marchandise, se sont révélés beaucoup plus forts que les risques auxquels il expose les grands trafiquants, lesquels trouvent dans la clandestinité, dans l'efficacité de leur machine organisationnelle internationale, dans leur réseau de complicités politiques et judiciaires, une garantie quasi totale d'impunité.

La criminalisation des drogues peut difficilement être évitée par qui n'est pas disposé à s'interroger sérieusement sur les raisons de la toxicomanie, ni à reconnaître ce qu'il y a de tragiquement vrai dans cette forme tourmentée de protestation sociale. Voir alors avant tout dans la toxicomanie une faute, signifie aggraver le conflit et l'absence de communication entre les jeunes et la société, laquelle, pré- 
cisément, les a poussés à cette forme ruineuse de recherche d'alternative.

Une autre mesure que notre société est amenée à prendre pour combattre la toxicomanie est l'internement forcé dans des communautés thérapeutiques dans lesquelles la méthode pratiquée pour le traitement du toxicomane fait appel dans une large mesure à la coercition. Cette mesure vise en définitive à guérir la toxicomanie en lui substituant d'autres formes de dépendance: au personnel professionnel, aux règles de la communauté, aux lois et à la culture de la société.

Quelle est l'efficacité d'une telle méthode ? Tout dépend de ce que l'on entend par ce terme. Si « efficace "signifie capable d'obtenir que les jeunes cessent de se droguer et rentrent dans la «vie normale ", il n'est pas exclu que dans un certain nombre de cas, la méthode fonctionne. Mais si "efficace" veut dire capable de résoudre le problème personnel et social suscité par la toxicomanie, ou même seulement de l'affronter, la réponse ne peut être que négative. La méthode autoritaire n'affronte pas le problème, mais le déplace. Elle ne met pas en question la culture qui a engendré la toxicomanie, mais la confirme.

\section{Alternative à la drogue et alternative à la société capitaliste}

Si ces analyses sont valables, la difficulté d'élaborer une stratégie efficace contre la drogue pour un système social qui y est aussi intimement lié devient plus évidente. Ces analyses peuvent même inciter à penser que cette bataille est perdue d'avance. D'autant plus qu'aujourd'hui le projet d'alternative à la société capitaliste est obsolète, dans la " gauche " elle-même.

En réalité, la drogue peut devenir un point de départ pour reformuler le problème de l'alternative en termes concrets et mobilisants. En effet, elle est indubitablement un de ces problèmes particuliers qui imposent à qui veut les affronter sérieusement, des analyses et des projets de caractère global. Ceci ne veut certes pas dire qu'une lutte efficace contre la drogue doit être renvoyée à la société future, mais que l'unique lutte véritablement efficace contre la drogue, aujourd'hui, est celle qui s'insère dans une bataille à long terme, pour une alternative sociale et culturelle. Cela signifie encore que les toxicomanes d'aujourd'hui sont eux-mêmes des sujets potentiels de cette bataille pour l'alternative.

Mais ne sont-ce pas là des hypothèses élaborées par quelque rêveur derrière sa table de travail ? Certes non. Ce sont, au contraire, 
des expériences d'une grande signification morale et politique, en cours depuis plusieurs années, en Italie et ailleurs, dont les protagonistes sont quelques communautés d'accueil. Expériences qui, bien que riches d'indications éducatives, culturelles et politiques, sont habituellement ignorées de la "gauche".

Certes, dans le cours du débat qui s'est récemment développé en Italie sur la riposte à la drogue, beaucoup se sont référés aux "communautés thérapeutiques", en voyant en elles l'unique réponse sérieuse au problème, et en demandant à l'État de les soutenir et de les renforcer. Mais un problème, par ailleurs décisif, tant du point de vue éducatif que politique, est resté dans l'ombre : celui de la différence de méthodes et d'objectifs entre les diverses communautés, de la distinction, en d'autres termes, entre les "communautés d'assistance » et les « communautés d'accueil ». Qu'est-ce qui démarque ces deux modèles?

Pour les "communautés d'assistance ", le drogué est essentiellement un malade, ayant besoin d'aide, incapable d'initiative, dont la guérison repose surtout sur l'intervention externe des experts auxquels on lui impose de se soumettre, en usant au besoin de moyens coercitifs. Une communauté enlignée sur un tel but devra être fortement hiérarchisée, c'est-à-dire axée sur une nette distinction entre "professionnels» et "usagers », "sains " et "malades», "normaux" et "anormaux ". Elle devra, en d'autres mots, être substantiellement homogène, structurellement et culturellement, à la société dans laquelle elle entend réintégrer les toxicomanes.

La méthode autoritaire qui s'y pratique devra réprimer ce sens critique, cette contestation de la société, ce refus de ses valeurs, qui font habituellement partie de l'itinéraire vers la drogue. Elle prépare donc des hommes et des femmes d'ordre, prêts à rentrer dans la "normalité ". Bien que se présentant, par conséquent, comme apolitique, cette éducation développe en réalité une action politique et morale, à l'enseigne de la restauration de l'ordre établi.

Le projet des « communautés d'accueil » naît, au contraire, d'une intuition : les toxicomanes sont non seulement des personnes en besoin d'aide, mais aussi des porteurs de potentialités insoupçonnées qui peuvent et doivent être mises en valeur. Ils sont donc appelés à être non seulement objets d'attention, mais encore sujets d'initiatives, de responsabilités, de création. Ils ne sont pas que des indigents à qui offrir des soins, mais aussi des personnes riches de valeurs et de sensibilité, souvent à un niveau exceptionnel, ellesmêmes capables de donner et de se donner. Et c'est justement dans la libération de ces énergies morales, affectives, intellectuelles, qu'est recherché le chemin plus authentique de la thérapie. La communauté tend donc, même si elle impose des règles de vie, à créer les 
conditions dans lesquelles chacun puisse élaborer et mettre en œuvre son projet de vie, découvrir et réaliser son identité. Ces conditions sont fournies par une vie en commun axée sur la confiance, la liberté, la participation, le partage.

Les deux attitudes face au problème inspirent en même temps deux orientations différentes dans l'analyse de sa genèse. Pour les uns, le problème prend sa source dans la transgression des règles morales et sociales, dans le refus des valeurs dominantes; pour les autres, au contraire, il découle précisément, dans une large mesure, de l'adhésion à ces valeurs et de la frustration qu'elles engendrent, c'est-à-dire de leur force répressive et marginalisatrice. Si dans la première perspective donc, les causes du problème sont recherchées uniquement dans la sphère personnelle et familiale, dans la seconde, la recherche s'oriente aussi, et indissociablement, vers le social et le politico-culturel. Ainsi, de façon inattendue, le malaise personnel devient un lieu privilégié pour analyser en profondeur la culture et la société, c'est-à-dire pour diagnostiquer le «malaise de la civilisation ".

Si donc la position assistancialiste se proclame habituellement apolitique, la position participative affirme, au contraire, un engagement politique explicite, orienté vers la construction d'une alternative de société. Si la communauté d'assistance est facilement tentée de devenir une oasis bienheureuse, un refuge où s'évader des contradictions de la société, la communauté participative se considère par nature une cellule vivante de l'ensemble social, dans lequel les contradictions sociales sont vécues avec une plus grande acuité et lucidité; où mûrissent l'exigence et l'espérance de les dépasser, en contribuant à la construction d'une humanisation différente; où l'expérience communautaire est vécue comme promesse et anticipation d'une convivialité humaine alternative.

\section{Conclusion}

L'expérience des communautés d'accueil, celles en particulier qui vivent de manière cohérente leur projet participatif, se révèle donc riche d'indications, en rapport soit à la lutte contre la drogue, soit à la bataille politique générale pour une alternative de société; la première de ces indications étant précisément la nécessaire interrelation entre les deux.

En ce qui concerne la lutte contre la drogue, les indications fournies par les communautés d'accueil ont trait aux analyses, au projet, à la stratégie. Sur le plan des analyses, elles invitent à rechercher l'origine de la toxicomanie dans l'interrelation entre 
facteurs personnels et sociaux, en attribuant une responsabilité particulière à la culture individualiste et compétitive, typique de la société capitaliste, et aux frustrations qu'elle engendre inévitablement dans la masse des jeunes. Pour cette raison même, elle suggère ensuite de relier les analyses psychosociales aux analyses structurelles traitant de l'interconnexion entre le marché de la drogue, l'économie capitaliste nationale et internationale, la machine étatique, le crime organisé.

Dans cette perspective, la libération de la toxicomanie implique aussi la libération de cette culture et, par conséquent, la maturation d'un "homme nouveau » et d'une "femme nouvelle » qui conçoivent leur épanouissement comme intimement lié à celui des autres, tous les autres, ceux en particulier, personnes, groupes, peuples, qui sont marginalisés par cette société. Un projet humain qui tende à se traduire en un projet anticapitaliste et anti-impérialiste de société. En définitive donc, la réponse au problème de la toxicomanie doit être recherchée sur le terrain où il se pose, quand on l'analyse en profondeur, c'est-à-dire celui du sens de la vie et de l'histoire.

La méthode proposée par les communautés d'accueil pour affronter ce problème est essentiellement celle de l'éducation libératrice : c'est-à-dire une formation à la liberté et à l'amour, à travers la pratique de la liberté et de l'amour, comprise concrètement comme participation et partage. Cette pratique, comme le projet qui l'inspire, dépasse cependant largement les limites de la communauté, pour se mesurer aux urgences de la libération de tous les marginalisés, au niveau national et international. Là où, par conséquent, l'engagement communautaire apparaît indissociable d'un engagement politique radical, d'une forte tension utopique. Dans la mesure aussi où les communautés d'accueil, à travers leur présence articulée sur le territoire, à travers leur réseau de parents, d'amis, de solidarités, deviennent des lieux de rassemblement, elles impliquent dans leur engagement social et politique des couches significatives de la population. Ainsi les toxicomanes, d'objets d'assistance deviennent sujets d'alternative; de marginalisés de cette société, protagonistes de la société future.

Ces indications pour la redéfinition de la lutte à la drogue sont sans doute très exigeantes et ne pourront pas être reçues par ceux qui ont décidé de contenir leur action dans les limites de compatibilité du système. Mais justement, la réflexion sur la drogue, menée à terme, se révèle un itinéraire privilégié pour démasquer le système et rendre manifeste son caractère suffocant, en particulier pour la jeunesse qui devrait y croître et s'y épanouir.

De telles indications devraient par contre être reçues et mises en valeur par les minorités qui n'ont pas renoncé à se battre pour 
I'alternative, qui ne se sont pas " repenties $»^{4}$. Peut-être en effet qu'une des raisons pour lesquelles la référence à l'alternative a perdu beaucoup de son mordant est qu'elle est perçue comme excessivement éloignée des batailles concrètes. Il s'agit alors de lui donner de nouvelles bases, à partir de tous ces problèmes sectoriels ressentis au niveau des masses, problèmes dont la solution se révèle impossible dans les limites de compatibilité du système capitaliste et doit, par conséquent, être recherchée dans une bataille globale pour l'alternative, par exemple le problème du chômage, du droit aux études, du droit à la santé, du désarmement, de l'environnement, etc. Par ce moyen, les couches de la population qui sont directement engagées dans la solution de ces problèmes particuliers devraient pouvoir être impliquées dans le mouvement pour l'alternative. Ainsi, pendant que la mobilisation sur les fronts sectoriels est alimentée et renforcée par la tension utopique, cette dernière, à travers sa présence diffuse dans les luttes sectorielles, acquiert concrétude, réalisme, force mobilisatrice.

\section{Notes}

${ }^{1}$ Note de l'auteur. La source principale de ces réflexions est constituée par quelques communautés d'accueil (San Benedetto al Porto, Gênes; Bergamasco et Frascaro, Alessandria; Ponzone, Acqui; Mignanego, Gênes), fondées et animées par D. Andrea Gallo, avec lesquelles je mène une enquête participative axée précisément sur leur expérience communautaire. Pour le moment cependant, en attendant la fin de la recherche, je présente cette lecture de l'expérience sous ma propre responsabilité.

J'ai de plus cherché à lier l'analyse psychosociale de la toxicomanie à l'analyse structurelle du marché de la drogue et, par conséquent, du phénomène de la mafia. Pour ces analyses, je me suis servi de quelques études publiées par la revue Segno di Palermo qui est, à ma connaissance, une des sources les plus riches d'information et d'analyse sur la mafia, perspicacement identifiée non comme un fait local et anormal, mais comme un phénomène organiquement lié au système capitaliste, sur le plan international, et révélateur de sa nature profonde.

Je voudrais en particulier attirer l'attention sur les numéros et articles suivants de cette revue : $n^{\text {os }} 31-32$, avril-mai 1982, "Droga. Dal ghetto all'impegno collettivo", et, dans celui-ci , l'article de U. Santino, "Economia e traffico della droga, mafia e organized crime " (p. 25-50); $n^{\circ} 27$, octobre-novembre 1981, "Violenza, mafia, morte », et, dans celui-ci, l'article de F. Renda, " La mafia nel secondo dopoguerra, un'interpretazione storiografica » (p. 77-107); enfin, $n^{\text {os }} 69-70$, avril-mai 1986, I'article de U. Santino, "La mafia finanziaria. Accumulazione illegale del capitale e complesso finanziario-industriale " (p. 7-49).

${ }^{2}$ Note du traducteur. Cet article a été publié en italien, sous le titre "La droga, fronte di lotta per l'alternativa ? ", dans : Animazione sociale, a. XIX, n० 14, 1989 : 45-53, et antérieurement dans: $A$ Sinistra, a. $I^{\circ}, n^{\circ} 1,1988$. Les lecteurs 
et lectrices d'ici qui seraient portés à voir dans l'analyse de Giulio Girardi une trop forte couleur italienne feraient bien de se rappeler qu'à la fin de 1989, la Banque Royale, la première en importance au Canada, demandait à I'Association des banquiers canadiens de "forcer toutés les banques du pays à prendre des mesures plus rigoureuses pour lutter contre le blanchissage de I'argent " (D. Francis, "Non au blanchiment de l'argent ", Le Soleil, samedi 16 décembre 1989: B-3). À la même époque, un rapport de police dénombrait neuf réseaux de drogue et 645 trafiquants, dans la seule ville de Québec, et dénotait " une augmentation de commerces qui ne servent qu'à blanchir l'argent provenant de la vente de stupéfiants " (N. Girard, "Selon un rapport de la SMQ, Québec compte de plus en plus de commerces pour blanchir l'argent », Journal de Québec, mardi 21 novembre 1989 : 2).

${ }^{3}$ N.d.t. Les " communautés d'accueil » constituent ce que nous appellerions ici des "ressources alternatives", dans lesquelles des intervenantes et intervenants partagent une expérience communautaire, impliquant pour plusieurs la vie en commun, avec des personnes de catégories sociales marginalisées, notamment toxicomanes et handicapé(e)s. Elles sont apparues et se sont développées à partir de la fin des années soixante, et on en trouve présentement dans plusieurs régions d'Italie. Un certain nombre d'entre elles, dont les plus anciennes, forment un réseau organisé à l'échelle nationale, le réseau de Capodarco. Les principes d'action qui les guident peuvent varier, mais plusieurs, comme l'explique Giulio Girardi, inscrivent leur pratique dans une recherche d'alternative de société.

${ }^{4}$ N.d.t. En italien contemporain, le terme " pentiti " (repentis) sert à désigner les terroristes qui renoncent à la lutte armée et choisissent de collaborer avec les autorités. Par extension, et dans le sens où il est employé ici, il désigne les militants et militantes politiques qui tournent le dos à leur option de gauche. 